\title{
Domain structure of the antiferromagnetic insulating state in $\mathrm{Nd}_{0.5} \mathrm{Sr}_{0.5} \mathrm{MnO}_{3}$
}

\author{
I. O. Shklyarevskiy, M. Yu. Shvedun, and S. L. Gnatchenko \\ Institute for Low Temperature Physics and Engineering of the National Academy of Sciences of Ukraine \\ 47 Lenin Ave., 61103 Kharkov, Ukraine \\ P. J. M. van Bentum, P. C. M. Cristianen, and J. C. Maan \\ Research Institute for Materials and High Field Magnet Laboratory, University of Nijmegen \\ Toernooiveld 1, NL-6525 ED Nijmegen, The Netherlands \\ K. V. Kamenev \\ Department of Physics and Astronomy, The University of Edinburg, Mayfield Road, Edinburg, EH9 3JZ, UK
}

\section{G. Balakrishnan and D. McK Paul}

Department of Physics, The University of Warwick, Coventry CV4 7AL, UK

Received June 21, 2001

\begin{abstract}
Optical reflectivity studies of the ferromagnetic metal (FMM) to antiferromagnetic insulator (AFI) phase transition are performed on $\mathrm{Nd}_{0.5} \mathrm{Sr}_{0.5} \mathrm{MnO}_{3}$ manganite in a wide temperature and magnetic field range. The formation of a domain structure in the AFI state during the FMM-AFI phase transition is observed. It is shown that the two types of domains observed are energetically equivalent states. On the basis of the experimental results and symmetry analysis we conclude that these domains are crystal twins. The twin domain structure of the AFI state in the $\mathrm{Nd}_{0.5} \mathrm{Sr}_{0.5} \mathrm{MnO}_{3}$ is visible in reflected unpolarized light due to a different tilting of the surface in the domains. The two-phase domain structure FMM+AFI formed in the vicinity of the phase transition is also studied. It is found that a thermodynamically equilibrium two-phase stripe domain structure does not develop. The absence of the magnetic intermediate state is due to the large energy of the interphase wall, which results in the stripe structure period being much larger than the size of the sample.
\end{abstract}

PACS: $75.30 . \mathrm{Kz}, 75.60 . \mathrm{Ch}$

\section{Introduction}

In the last decade the perovskite manganese oxides with the basic formula $\mathrm{R}_{1-x} \mathrm{~A}_{x} \mathrm{MnO}_{3}$ (where $\mathrm{R}=$ trivalent rare-earth ion, $\mathrm{A}=$ divalent alkaline ion) have been of great interest because of the unusual magnetic and transport properties [1]. The most spectacular effect in the manganites is the colossal magnetoresistance observed in the vicinity of the Curie temperature. Moreover these compounds exhibit interesting phenomena such as charge and orbital ordering, the Jahn-Teller effect, and magnetic- or electric-field-induced phase transitions.
In some of them a spontaneous and magnetic-fieldinduced phase transition from an antiferromagnetic insulating (AFI) to a ferromagnetic metallic (FMM) state takes place [2-4]. The nature of this phase transition is rather complicated and its understanding is yet to emerge.

$\mathrm{Nd}_{0.5} \mathrm{Sr}_{0.5} \mathrm{MnO}_{3}$ is one of the manganites that demonstrate such a FMM-AFI phase transition [2-4]. The ferromagnetic ordering occurs in this crystal at $T_{C} \approx 255 \mathrm{~K}$. At a temperature $T_{M-I}$ near $160 \mathrm{~K} \mathrm{a}$ spontaneous first-order FMM-AFI phase transition takes place. This transition is accompanied by charge ordering, i.e., spatial ordering of the holes and 
corresponding spatial ordering of the $\mathrm{Mn}^{3+}$ and $\mathrm{Mn}^{4+}$ ions. In the temperature range $T<T_{M-I}$ the application of a magnetic field leads to melting of the charge order in $\mathrm{Nd}_{0.5} \mathrm{Sr}_{0.5} \mathrm{MnO}_{3}$ and induces a transition to the FMM state [2]. The electric conductivity during this transition varies over two orders of magnitude, and the magnetization changes by a value of $2.5 \mu_{B}$ per $\mathrm{Mn}$ atom [2]. The FMM-AFI phase transition in $\mathrm{Nd}_{0.5} \mathrm{Sr}_{0.5} \mathrm{MnO}_{3}$ is also accompanied by considerable changes in the lattice parameters $\left(\sim 10^{-3}-10^{-2}\right)$ and unit cell volume $\left(\sim 10^{-3}\right)$ [2,5]. It was suggested in early studies [2] that there is no change in the crystal symmetry at this transition (the crystal has orthorhombic symmetry both in the FMM and AFI states). However, recent synchrotron and neutron diffraction investigations have shown that the FMM-AFI transition is accompanied by a lowering of the crystal symmetry from orthorhombic (space group Imma) to monoclinic (space group $P 2_{1} / m$ ) [5,6]. In a previous paper [7] we reported that the FMM-AFI phase transition in $\mathrm{Nd}_{0.5} \mathrm{Sr}_{0.5} \mathrm{MnO}_{3}$ is followed by the formation of a domain structure in the AFI state which is visible in unpolarized white light.

In the present study we have investigated this domain structure in a wide temperature and magnetic field range with the goal of understanding its nature.

\section{Experiment}

The $\mathrm{Nd}_{0.5} \mathrm{Sr}_{0.5} \mathrm{MnO}_{3}$ crystal was melt-grown by standard floating zone method and had a cylindrical form $3 \mathrm{~mm}$ in diameter. X-ray analysis showed it to be a single crystal. The samples were then cut perpendicular to the cylinder axis; their thickness varied from 0.5 to $3 \mathrm{~mm}$. For our experiments the samples were optically polished with diamond paste. To remove the surface strain that occurs as a result of polishing they were annealed for 20 hours at $950{ }^{\circ} \mathrm{C}$ in air.

The experiments were performed on two setups. The first one permits simultaneous optical imaging of the domain structure and indirect measurements of the reflected light intensity from the different domains. The samples were mounted in a continuous flow He cryostat placed in a Bitter magnet with magnetic field range up to $15 \mathrm{~T}$. The image of the sample produced by the reflectance optical microscope was recorded with a CCD camera connected to a video recorder. Nonpolarized white light from a filament lamp was used to image the surface of the sample. The video images were subsequently analyzed to determine the reflected light intensity. In this case an interference filter with $\lambda=634 \mathrm{~nm}$ was placed after the source of light. Field dependences of the reflected light intensity were obtained by computer processing of the image.

The second setup allows direct measurement of the reflected light intensity. In this case the sample was mounted in an optical He cryostat, where it was placed in a superconducting magnet with a maximum field of $5 \mathrm{~T}$. A He-Ne laser at $\lambda=633 \mathrm{~nm}$ was used as a source of light with an optical chopper in the beam. The light reflected from the sample was detected with a photomultiplier connected to a lock-in. This setup as well as the previous one allows us to measure the reflected light intensity both from the whole surface of the sample and from separate domains. To measure the intensity of the light reflected from individual domains the image of the sample was projected on a screen with a diaphragm. By shifting the diaphragm position we could measure the reflected light intensity from different domains. This setup also affords the possibility of visual observation of the domain structure using a reflectance optical microscope and video camera.

The magnetization measurements were carried out with a moving-sample magnetometer in a variable-temperature cryostat and in fields up to $20 \mathrm{~T}$.

\section{Experimental results}

It was found that there are distinct changes in the unpolarized reflected light intensity at the FMM-AFI phase transition in $\mathrm{Nd}_{0.5} \mathrm{Sr}_{0.5} \mathrm{MnO}_{3}$ [7]. This effect made possible the optical observation of the two-phase domain state that is formed at this first-order phase transition. Besides, the domain structure in the AFI phase was visually observed and recorded. With temperature decrease a spontaneous FMM-AFI phase transition takes place at $T \approx 154 \mathrm{~K}$, while temperature increase results in a reverse transition at $T \approx 162 \mathrm{~K}$. Thus the first-order FMM-AFI phase transition is accompanied by substantial hysteresis. Coexistence of the FMM and AFI phases was observed in a temperature region of about $2 \mathrm{~K}$. The AFI phase was nonuniform with clearly visible domain structure that remained unchanged in the whole temperature range $(20-160 \mathrm{~K})$ at zero magnetic field. There were also no variations of the domain structure in a magnetic field up to the field of the phase transition to the FMM state. After a spontaneous or field-induced phase transition to the FMM state and a subsequent reverse transition to the AFI state the newly formed domain structure was usually different from the previous one. From this fact one can conclude that the domain structure in the AFI state emerges at the 

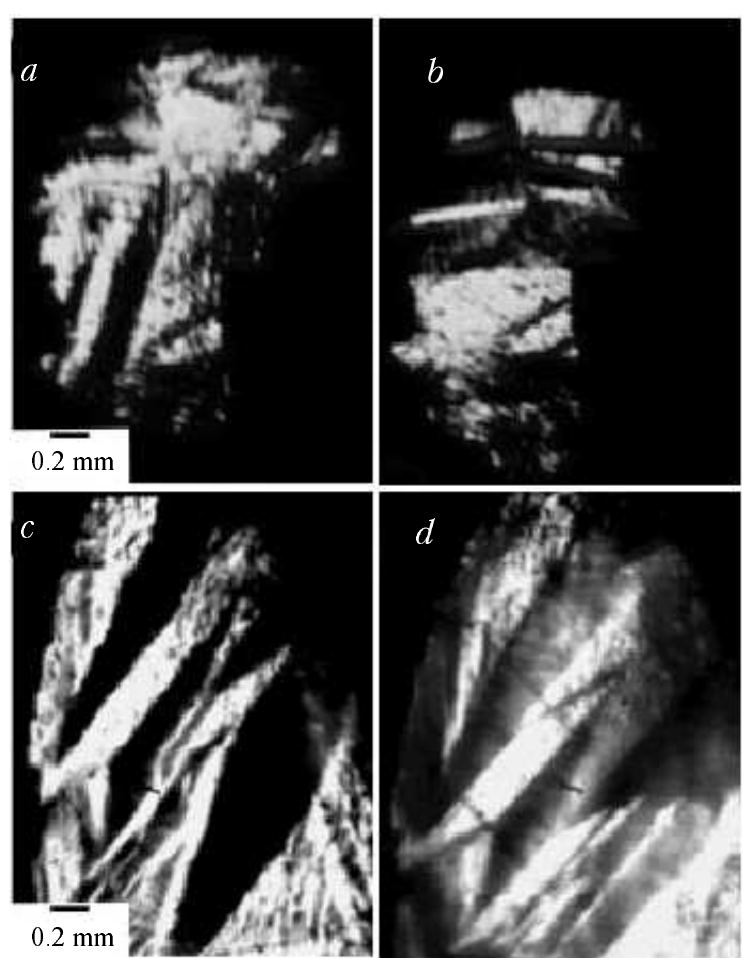

Fig. 1. Domain structure of two $\mathrm{Nd}_{0.5} \mathrm{Sr}_{0.5} \mathrm{MnO}_{3}$ crystals in the AFI state. Images $(a)$ and $(b)$ show the domain structure after the first and second AFI-FMM-AFI transition cycles, respectively. Images $(c)$ and $(d)$ show a similar sequence for a second crystal.

FMM-AFI phase transition. This domain structure does not exist in the FMM state.

The photos in Fig. 1 show the domain structure of $\mathrm{Nd}_{0.5} \mathrm{Sr}_{0.5} \mathrm{MnO}_{3}$ in the AFI state. Two examples are given in Fig. 1, showing the domain structure of the same sample after the first (Fig. 1,a) and second (Fig. 1,b) AFI-FMM-AFI transition cycle. A comparison of the two images shows that the domain structures formed in subsequent cycles can be quite different. The images in Fig. 1,c and
Fig. 1, $d$, respectively, represent a similar sequence on a second sample.

The main features of the domain structure observed in the AFI state are the following. Two types of domains (bright and dark) exist in the AFI state. The domain walls have a favorable orientation (Fig. 1). Two primary directions along which the walls are oriented can be selected in the sample (see Fig. 1,a,b).

Figure 2 shows the representative stages in the domain formation during the FMM-AFI phase transition. Figure 2, $a$ shows an image of the sample in the homogeneous FMM phase. The transition occurs by the nucleation and subsequent propagation of the domains of the energetically favorable phase. As these domains extend, the concentration of the energetically unfavorable phase decreases, until the sample is completely transformed to the AFI phase. Figure 2,b represents an image of a two-phase state with both FMM and AFI phases present. Figure 2,c is an image of the surface in the fully developed AFI phase but with clear optical contrast in bright and dark domains. We did not find an intermediate state with a periodic FMMAFI domain structure in the $\mathrm{Nd}_{0.5} \mathrm{Sr}_{0.5} \mathrm{MnO}_{3}$ crystals.

Field dependences of the reflected light intensity $I(H)$ from the whole surface of the sample were measured to determine the hysteretic AFI-FMM transition fields and the field range where AFI and FMM phases coexist. Typical $I(H)$ curves are shown in Fig. 3, $a$. The reflected light intensity is given in arbitrary units and normalized on the value of the reflected light intensity in the FMM state. The field at which the jump in intensity occurs corresponds to the AFI-FMM phase transition field. The transition field decreases with temperature increase. It is clearly seen that this first-order phase transition is accompanied by a hysteresis in $I(H)$. This hysteresis
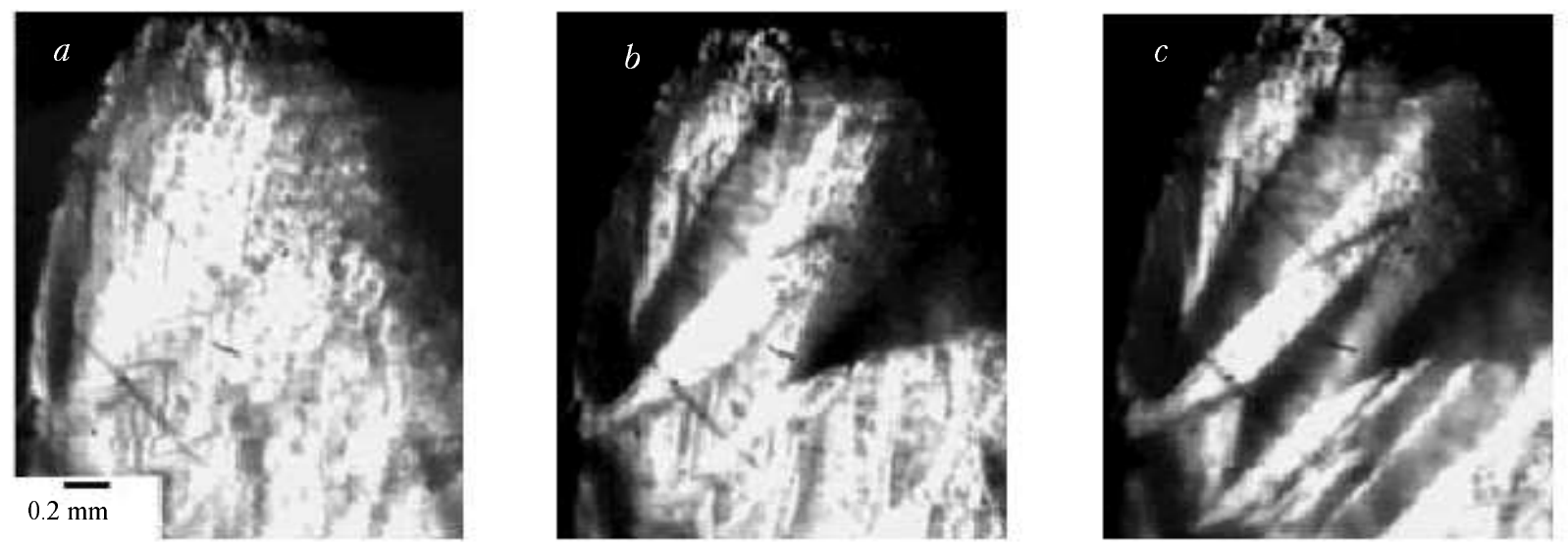

Fig. 2. Images of the field-induced FMM-AFI phase transition in $\mathrm{Nd}_{0.5} \mathrm{Sr}_{0.5} \mathrm{MnO}_{3}$. The temperature of the sample $T=150 \mathrm{~K}$. External magnetic field $H, \mathrm{~T}: 1.8(a), 1.2(b), 0(c)$. 
becomes smaller as the temperature increases. The width of the temperature range of the AFI-FMM phase coexistence also decreases at higher temperatures. The transition width decreases from $\Delta H_{\text {coex }} \approx 1 \mathrm{~T}$ at $T=20 \mathrm{~K}$ to $\Delta H_{\text {coex }} \approx 0.5 \mathrm{~T}$ at $T=$ $=140 \mathrm{~K}$. The change in the reflected light intensity level in the AFI state after a full cycle can be attributed to a different concentration of the bright and dark AFI domains before and after the AFIFMM-AFI transition. Note that the reflected light intensity in the FMM state remains practically unchanged (within the experimental error limits $\pm 5 \%)$ in the present temperature and magnetic field range.

For comparison we measured the field dependences of the magnetization $M(H)$ for the same sample. Typical $M(H)$ curves are shown in Fig. 3,b. The width of the phase transition on the $M(H)$ curves is generally larger than that seen on the optical $I(H)$ curves. This indicates some additional inhomogeneity of the bulk of the sample.

To reveal the nature of the domain structure observed in the AFI state the field dependences of the reflected light intensity were also measured for single domain areas of the sample. Figure 4 shows the $I(H)$ curves for dark $(a)$ and bright $(b)$ do-

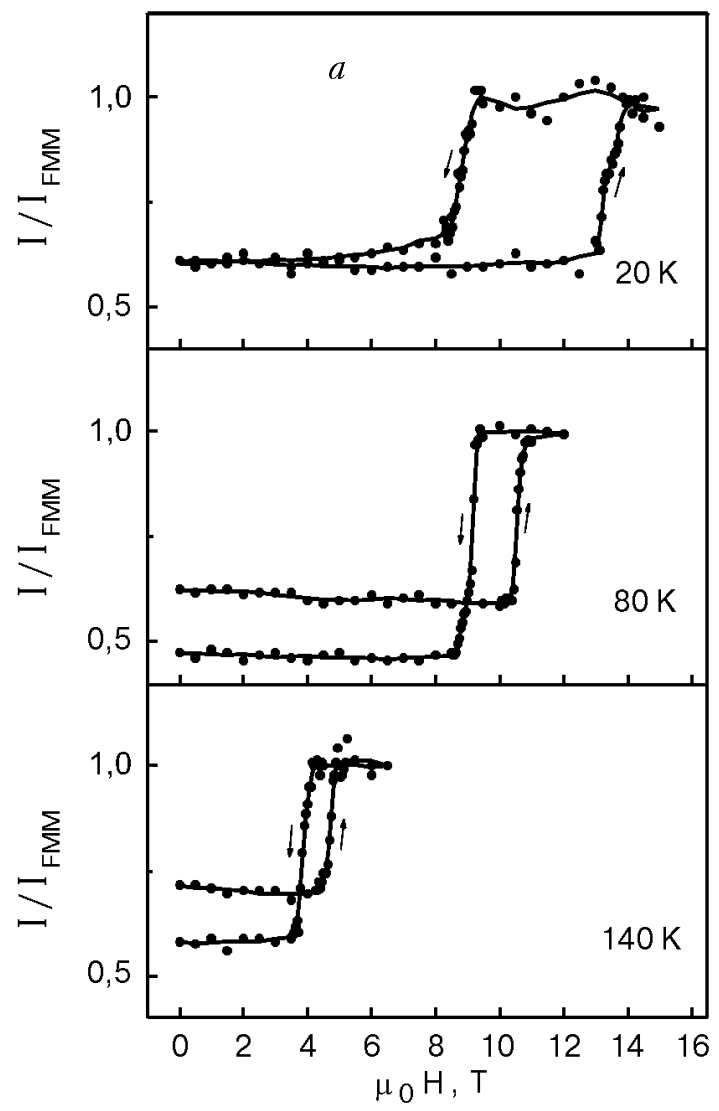

mains at different temperatures. The reflected light intensity for dark domains is much lower than for the FMM state while for bright domains it is higher. It is important to note that the AFI-FMM phase transition occurs at the same magnetic field in the dark and bright domains. Therefore these domains are not thermodynamically different phases but energetically equivalent states. The strongest drop of the reflected light intensity was observed in the $I(H)$ curves for bright domains in the vicinity of the phase transition (see Fig. 4,b, bottom curve). Such behavior of the intensity can be associated with changes in the optical properties of the crystal as a result of the elastic strains arising near the interphase wall. These elastic strains undoubtedly must arise because of the large changes in the crystal lattice parameters at the phase transition $[2,5]$.

As was mentioned above, the domain structure in the AFI state before transition to the FMM state and after reverse transition to the AFI state often differs. Therefore $I(H)$ curves like those presented in Fig. 5 were observed in some cases. Figure 5, $a$ shows an $I(H)$ curve that corresponds to the transition from a dark AFI domain to the FMM state and the inverse transition to a bright AFI domain. In Fig. $5, b$ the $I(H)$ dependence that corresponds to

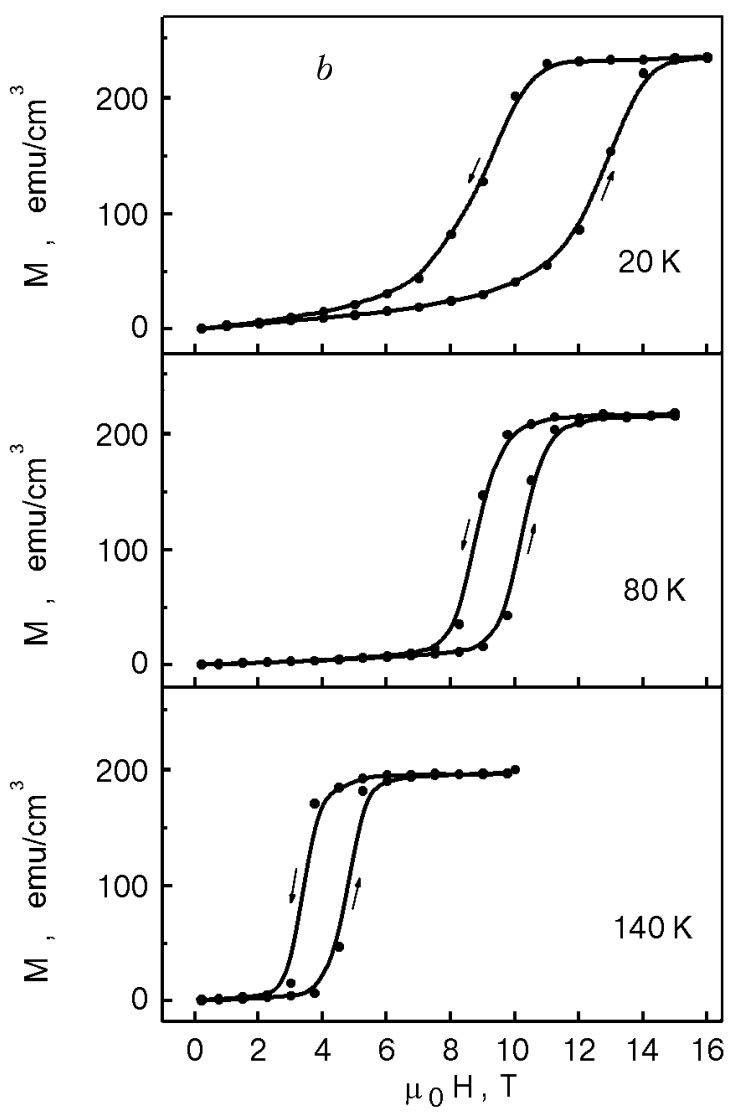

Fig. 3. Field dependences of the integrated reflected light intensity for the whole surface of the sample ( $a$ ), and field dependences of the magnetization $(b) . I(H)$ and $M(H)$ curves are presented for $T=20,80$ and $140 \mathrm{~K}$. The reflectivity is normalized to the FMM state. 

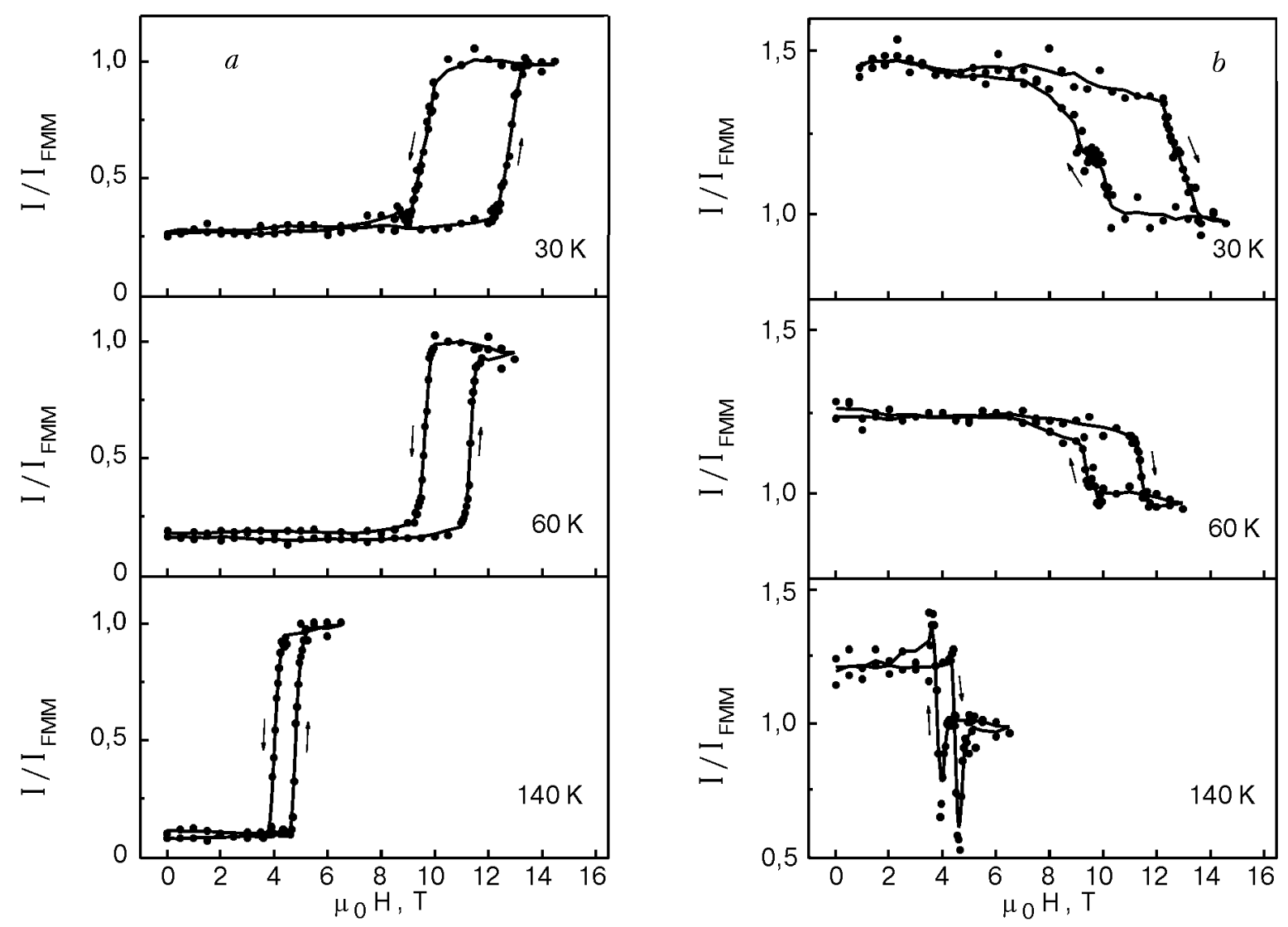

Fig. 4. Field dependences of the reflected light intensity measured for dark (a) and bright (b) domains at the temperatures 30 , 60, and $140 \mathrm{~K}$.

the transition from a bright AFI domain to the FMM state and the inverse transition to a dark AFI state is shown.

The $H-T$ phase diagram of $\mathrm{Nd}_{0.5} \mathrm{Sr}_{0.5} \mathrm{MnO}_{3}$ constructed from the results of the optical studies is shown in Fig. 6. The value of the transition fields in the phase diagram were determined from the $I(H)$ curves for the whole sample and correspond to the center of the field interval of the AFI+FMM phase coexistence. The unfilled symbols correspond to the AFI-FMM phase transition observed with increasing magnetic field and the filled symbols correspond to the reverse transition in decreasing field. The difference between the transition fields obtained for these two cases corresponds to the hysteresis in the $I(H)$ curves. It is clearly seen in Fig. 6 that the hysteresis decreases at higher temperatures.

Another sets of symbols presents the transition field values determined from the $I(H)$ dependences measured separately for dark and bright domains. The phase diagram clearly demonstrates good coincidence in the transition fields for dark and bright domains in the whole temperature range.
The values of the transition fields obtained from the $M(H)$ curves are also indicated in the phase diagram (Fig. 6) for comparison with the optical

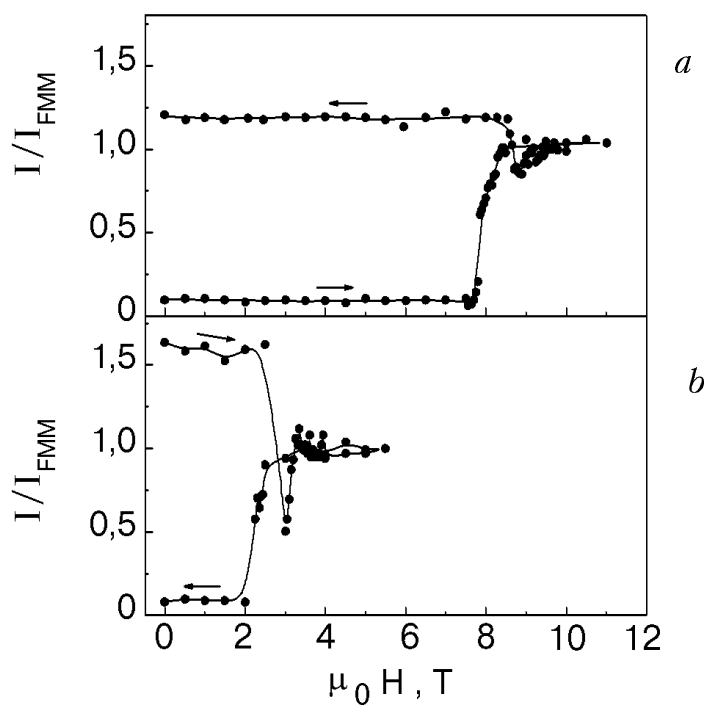

Fig. 5. Field dependences of the reflected light intensity measured in a single domain area of the sample for the cases when after an AFI-FMM-AFI transition cycle at the place of the dark domain a bright one arises, at $T=100 \mathrm{~K}(a)$, and vice versa, at $T=150 \mathrm{~K}(b)$. 


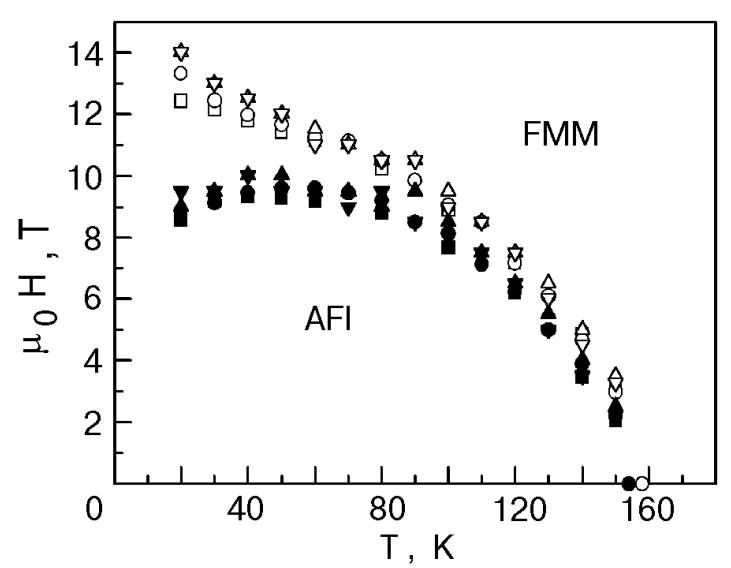

Fig. 6. $H-T$ phase diagram of $\mathrm{Nd}_{0.5} \mathrm{Sr}_{0.5} \mathrm{MnO}_{3}$ constructed from optical studies. The unfilled symbols correspond to the phase transition from the AFI to the FMM state and filled symbols correspond to the reverse transition from the FMM to the AFI state. The transition fields were determined from the $I(H)$ dependences for the whole sample (circles) and for the bright (up triangles) and dark (down triangles) domains, and also from the $M(H)$ dependences (squares).

results. These values were determined in the same way as the transition fields from $I(H)$ curves. The results obtained from optical and magnetic measurements are in satisfactory agreement. Therefore optical studies probe information about a rather deep surface layer of the sample and represent the bulk properties of the crystal. The influence of the surface on the AFI-FMM transition in $\mathrm{Nd}_{0.5} \mathrm{Sr}_{0.5} \mathrm{MnO}_{3}$ was not revealed.

The above experiments demonstrate that the two types of domains (dark and bright) observed in the AFI phase are energetically equivalent states formed during the transition from the FMM phase to the AFI phase. It is reasonable to suggest that these domains are crystal twins emerging at the FMMAFI phase transition, accompanied by a lowering of the crystal symmetry from orthorhombic to monoclinic. However, the problem of the optical contrast in unpolarized reflected light at the crystal twins still remains to be solved.

During visual observation of the domain structure in the AFI state we found that variation of the angle of incidence of light on the sample results in changes of the optical contrast between domains. Dark domains can transform to bright or vice versa, and at certain angle of incidence the optical contrast between domains may disappear. Moreover, the contrast between domains also vanishes when a microscope objective with a sufficiently large aperture is used. This suggests that during crystal twin formation the surface of the sample becomes corrugated and the surfaces of the twins of different type form a certain angle with each other. In this case the light intensities reflected from the surfaces of the different types of twins and captured by the microscope objective can be different, which results in an optical contrast between domains. In other words, part of the light reflected from the surface of the twins of one type is not captured by the objective and these domains are observed as dark.

To check this suggestion the following experiment was performed. The basic scheme of the optical setup used for this experiment is presented on the upper panel of Fig. 7. A beam from a $\mathrm{He}-\mathrm{Ne}$
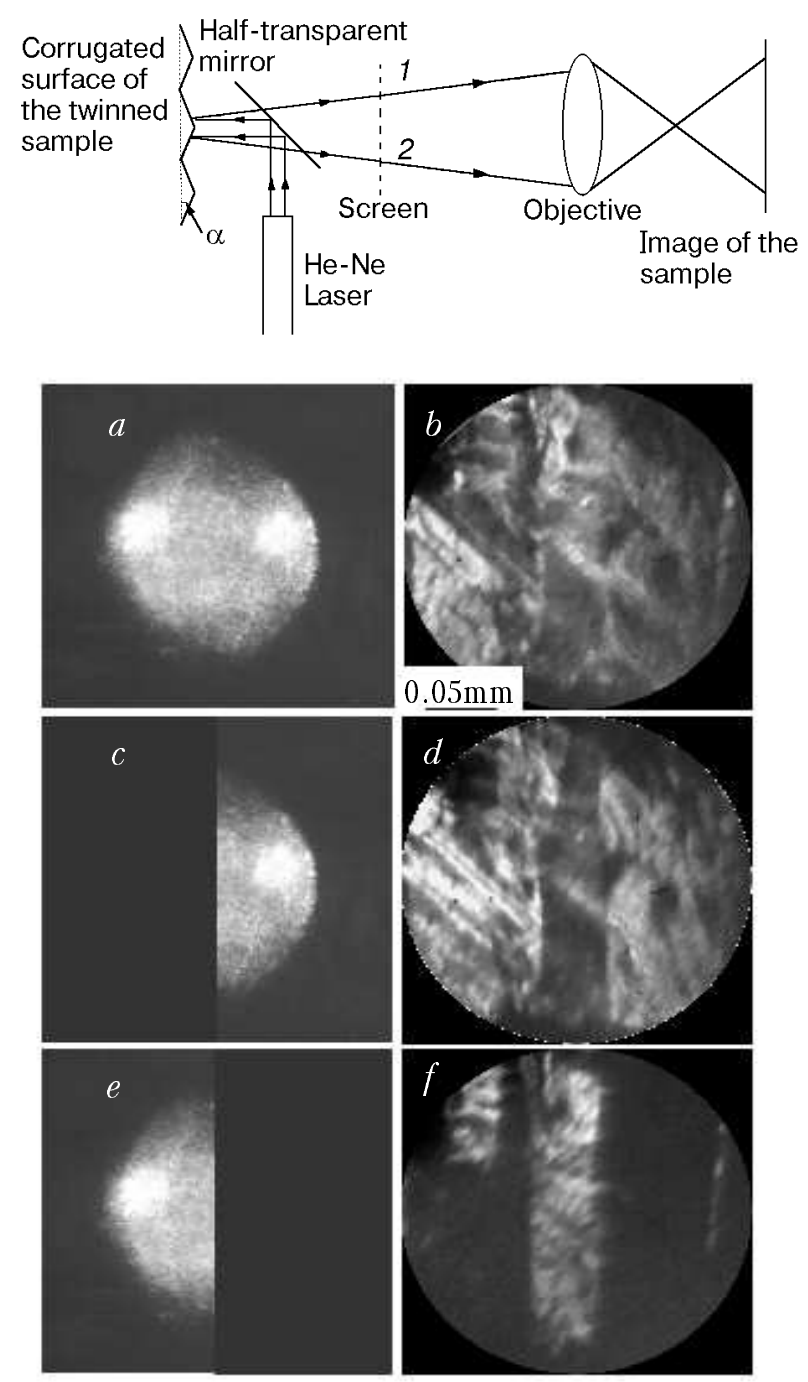

Fig. 7. (Upper panel) The optical setup to demonstrate the origin of the optical contrast between crystal twins. (Lower panel) a) - Two white spots on the screen correspond to separate major directions for specular reflection from the corrugated surface of the sample. $b$ ) - Image of the sample, where both reflected beams are captured by the objective. No domain structure is observed. $c$ ) and $d$ ): The first beam is closed off. The domain structure is observed visually. $e$ ) and $f$ ): The second beam is closed. Inverse changes occur in the contrast of the domain structure. 
laser illuminates an area of the sample including both types of domains. The reflected light is used to image the surface using a microscope objective. On an intermediate screen we can determine the intensity distribution in the reflected beam. In the FMM phase we observe a single bright spot on the screen, corresponding to the specular reflection of the optically polished surface. However, in the AFI domain phase we find two spots (Fig. 7,a). If the optical alignment is such that the light of both spots is used in the image, then no optical contrast is seen between the various domains (see Fig. 7,b). However, if we close off half of the beam with one of the two bright spots (Fig. 7,c and $e$ ), we can observe the contrast domain structure (Fig. $7, d$ and $f$ ). This strongly suggests that the optical contrast between the domains is caused by a different angle of specular reflection for the two types of domains. The estimated tilt angle of the domain surface is $\alpha \approx 1^{\circ}$.

This experiment allows not only to explain the existence of the optical contrast, but also to correct the data for $I(H)$. The real change of the reflected light intensity during the AFI-FMM phase transition can be observed only on the $I(H)$ curves for bright domains. A small increase of the reflected light intensity, i.e., a small increase of the reflection coefficient, was observed on these curves at the transition to the AFI phase (Fig. 4,b). The decrease in the intensity of the $I(H)$ curves for dark domains and for the whole sample is related to the fact that part of the reflected light was not captured by the objective and registered by the photomultiplier. Thus the curves for dark domains and for the whole sample did not show the real picture of the changes in the reflectance at the transition. However, they gave a much better possibility than $\operatorname{did} I(H)$ for the bright domains for determining of the transition field value and the region of the phase coexistence.

\section{Discussion}

As we have said, the cause of the AFI phase inhomogeneity can be the formation of a twin domain structure during the transition to a state with lower crystal symmetry. According to [5,6,8], the symmetry of $\mathrm{Nd}_{0.5} \mathrm{Sr}_{0.5} \mathrm{MnO}_{3}$ changes from orthorhombic (space group Imma) to monoclinic (space group $\left.P 2_{1} / m\right)$ at the FMM-AFI transition. The formation of the twin domain structure in the AFI state previously was observed in $\mathrm{Pr}_{0.5} \mathrm{Sr}_{0.5} \mathrm{MnO}_{3}$ by electron diffraction techniques [9].

Let us perform a symmetry analysis of the twin domain structure that can arise in the asymmetric phase during the phase transition accompanying the lowering of the symmetry from orthorhombic (point group $\mathrm{mmm}$ ) to monoclinic (point group $2 / \mathrm{m}$ ). At the structural transition the crystal loses the following symmetry operations: two reflection planes $2_{x}$, $2_{y}$ and two rotation axes $2_{x}$ and $2_{y}$ (the monoclinic axis is the $z$ axis). The lost symmetry operations are indeed twinning operations. Two types of crystal twins can be formed at this phase transition. These twins differ by the sign of the shear deformation in the $x y$ plane and transform one into another by the twinning operations. Monoclinic shear deformations in the two types of twins can be described by the second-rank tensors $\varepsilon_{i j}^{+}$and $\varepsilon_{i j}^{-}$with the following matrices:

$$
\varepsilon_{i j}^{+}=\left(\begin{array}{lll}
\varepsilon_{11} & \varepsilon_{12} & 0 \\
\varepsilon_{21} & \varepsilon_{22} & 0 \\
0 & 0 & \varepsilon_{33}
\end{array}\right), \varepsilon_{i j}^{-}=\left(\begin{array}{ccc}
\varepsilon_{11} & -\varepsilon_{12} & 0 \\
-\varepsilon_{21} & \varepsilon_{22} & 0 \\
0 & 0 & \varepsilon_{33}
\end{array}\right) .
$$

Consider possible coherent (without any twinning dislocations) walls between these twins. The condition of coexistence of the deformations, i.e., the absence of strains in the wall, is [10]

$$
\left(\varepsilon_{i j}^{+}-\varepsilon_{i j}^{-}\right) x_{i} x_{j}=0 .
$$

In order for (2) to be an equation of a plane the following additional condition must be satisfied:

$$
\operatorname{det}\left|\varepsilon_{i j}^{+}-\varepsilon_{i j}^{-}\right|=0 .
$$

It is easy to check that in the case considered, the condition (3) is satisfied and the solutions of the equation (2) are two orthogonal planes $x=0$ and $y=0$. Thus coherent domain walls have to be arranged in these crystal planes.

When the results of the above analysis are compared with the experimental data the following can be seen. The analysis performed shows that two types of crystal twins can arise at the $\mathrm{mmm} \rightarrow 2 / \mathrm{m}$ phase transition. This is consistent with the optical observations that also show two types of domains in the AFI state of $\mathrm{Nd}_{0.5} \mathrm{Sr}_{0.5} \mathrm{MnO}_{3}$. It also follows from the symmetry analysis that coherent walls of two types (planes $x=0$ and $y=0$ ) occur in the twinned crystal. It is clearly seen from Fig. 1,a,b that there are two favorable directions of orientation of the domain walls. The slight deviations of the domain walls from these directions can be attributed to the elastic strains always existing in a real crystal. The angle between the two prevalent orientations of the domain walls is $70-80^{\circ}$ (Fig. $1, a$ ). If the monoclinic $z$ axis is perpendicular to the surface of the sample, then the angle between coherent domain walls oriented in the $x=0$ and 
$y=0$ planes is equal to $90^{\circ}$. However, if the monoclinic axis is tilted away from the normal to the surface of the sample, as is confirmed by $\mathrm{x}$-ray analysis of our samples, this angle is less, and that explains why the angle between domain walls observed in the experiment differs from $90^{\circ}$.

Thus the results of the symmetry analysis are in good agreement with the experimental data. This allows us to conclude that the domains observed in the AFI state are crystal twins that form at the transition from the orthorhombic FMM phase to the monoclinic AFI phase.

Finally, let us consider some properties of the two-phase state observed near the FMM-AFI phase transition. As was mentioned above, no magnetic intermediate state, i.e., thermodynamically stable periodic two-phase domain structure, forms at this transition. Usually the absence of the intermediate state can be attributed to variability of the transition field in the sample. The intermediate state does not arise if this variability substantially exceeds the value $4 \pi N \Delta M$, where $\Delta M$ is the magnetization jump at the phase transition and $N$ is the demagnetizing factor. Typically for $\mathrm{Nd}_{0.5} \mathrm{Sr}_{0.5} \mathrm{MnO}_{3}$ we find $\Delta M=$ $=200-220 \mathrm{emu} / \mathrm{cm}^{3}$ (Fig. 3,b) and a field interval of the intermediate state $\Delta H=0.3-0.4 \mathrm{~T}$. This indicates that the absence of the intermediate state is not due to inhomogeneity of the sample.

Apparently a large energy of the interphase wall caused by the strong elastic strains is the main reason why the intermediate state does not form. These elastic strains must arise in the vicinity of the interphase wall as a result of the dramatic changes $\left(\varepsilon \approx 1 \cdot 10^{-2}\right)$ [4] in the crystal lattice parameters at the FMM-AFI phase transition. Optical measurements in polarized light give us the possibility of estimating the width of the strained area of the crystal in the wall vicinity $l \approx 60 \mu \mathrm{m}$. Assuming that the dominant contribution to the interphase wall energy is the contribution from the elastic strains, the wall energy can be estimated using the following formula

$$
\sigma=C \varepsilon^{2} l
$$

where $C$ is the elastic constant. For this rough estimation we have neglected the strain inhomogeneity in the vicinity of the wall. Assuming $C \sim$ $\sim 10^{12} \mathrm{erg} / \mathrm{cm}^{3}$, we get $\sigma \approx 6 \cdot 10^{5} \mathrm{erg} / \mathrm{cm}^{2}$. Using the value obtained for $\sigma$, we can estimate the period $D$ of the stripe domain structure of the magnetic intermediate state [11]:

$$
D=\left(\frac{\sigma d}{F(\xi)(\Delta M)^{2}}\right)^{1 / 2},
$$

where $d$ is the sample thickness, $F(\xi)=1.71 / 4$ for $\xi=0.5[11], \xi$ is the concentration of the AFI and FMM phases. For $d=1 \mathrm{~mm}$ and $\Delta M=210 \mathrm{emu} / \mathrm{cm}^{3}$ we get $D \approx 2 \mathrm{~cm}$. Thus the estimated period of the equilibrium stripe two-phase domain structure substantially exceeds the size of the sample. This explains the absence of the intermediate state at the FMM-AFI phase transition in our experiments.

\section{Conclusions}

The optical studies have shown that a twin domain structure arises in the AFI state as a result of the lowering of the crystal symmetry at the FMM-AFI phase transition. The crystal twins could be imaged in unpolarized light. It was shown that the cause of the optical contrast between the two types of twins is the local tilting of the surface of the twin domains. As far as we know, a twin domain structure has not been observed earlier by this method. Note that the existence of the twin domain structure was confirmed by direct $\mathrm{x}$-ray studies [8].

It was also shown that no thermodynamically stable striped two-phase domain structure forms at the FMM-AFI phase transition. The absence of the magnetic intermediate state at this transition is due to the large energy of the interphase wall, which results in a stripe domain periodicity much larger than the size of the sample.

1. A. P. Ramires, J. Phys. Condens. Matter 9, 8171 (1997).

2. H. Kuwahara, Y. Tomioka, A. Asamitsu, Y. Moritomo, and Y. Tokura, Science 270, 961 (1995).

3. Y. Tokura, H. Kuwahara, Y. Moritimo, Y. Tomioka, and A. Asamitsu, Phys. Rev. Lett. 76, 3184 (1996).

4. H. Kawano, R. Kajimoto, H. Yoshizawa, Y. Tomioka, H. Kuwahara, and Y. Tokura, Phys. Rev. Lett. 78, 4253 (1997).

5. R. Mahendrian, M. R. Ibarra, A. Maignan, F. Millange, A. Arulraj, R. Mahash, B. Raveau, and C. N. R. Rao, Phys. Rev. Lett. 82, 2191 (1999).

6. P. M. Woodward, D. E. Cox, T. Vogt, C. N. R. Rao, and A. K. Cheetham, Chem. Mater. 11, 3528 (1999).

7. S. L. Gnatchenko, A. B. Chizhik, I. O. Shklyarevskiy, D. N. Merenkov, V. I. Kamenev, Yu. G. Pashkevich, K. V. Kamenev, G. Balakrishnan, and D. McK Paul, Fiz. Nizk. Temp. 25, 992 (1999) [Low Temp. Phys. 25, 744 (1999)].

8. V. Eremenko, S. Gnatchenko, N. Makedonskaya, Yu. Shabakayeva, M. Shvedun, V. Sirenko, J. Fink-Finowicki, K. V. Kamenev, G. Balakrishnan, and D. McK Paul, Fiz. Nizk. Temp. 27, 1258 (2001).

9. F. Damay, C. Martin, M. Hervieu, A. Maignan, B. Raveau, G. Andre, F. Bouree, J. Magn. Magn. Mater. 184, 71 (1998).

10. J. Sapriel, Phys. Rev. B12, 5128 (1975).

11. V. G. Baryakhtar, A. E. Borovik, and V. A. Popov, $Z h$. Eksp. Teor. Fiz. 62, 2233 (1972). 